\title{
Glutamate receptor-mediated ischemic injury of premyelinated central axons.
}

\begin{tabular}{|c|c|}
\hline Journal: & Annals of Neurology \\
\hline Manuscript ID: & ANA-09-0029.R1 \\
\hline Wiley - Manuscript type: & Research Article \\
\hline $\begin{array}{r}\text { Date Submitted by the } \\
\text { Author: }\end{array}$ & \\
\hline Complete List of Authors: & $\begin{array}{l}\text { Alix, James; University of Liecester, Cell Physiology and } \\
\text { Pharmacology } \\
\text { Fern, Robert; University of Liecester, Cell Physiology and } \\
\text { Pharmacology }\end{array}$ \\
\hline Domain: & Child Neurology \\
\hline Keywords: & cerebral palsy, ischemia, axon \\
\hline
\end{tabular}


Glutamate receptor-mediated ischemic injury of premyelinated central axons.

James J. P. Alix and Robert Fern

Department of Cell Physiology and Pharmacology, University of Leicester, Leicester,

UK.

Short Title: Ischemic injury of developing central axons

Key Words: Axon; Ischemia; Glutamate

Deleted: +2

Figures: 7 (+5 supplemental Figures)

Words: 2999

(2. - n

Deleted: 2900I

Pages: 21

Corresponding Author:

Dr Bob Fern

Department of Cell Physiology \& Pharmacology, University of Leicester, P.O.

Box 138, University Road, Leicester, LE1 9HN, England.

Telephone: +44 (0) 1162523098

Fax: +44 (0) 1162525045 


\begin{abstract}
Objective: Ischemic injury of axons is a feature of periventricular leukomalacia, a pathological correlate of cerebral palsy. Recent evidence suggest that axons are damaged before they receive the first layer of compact myelin. We here examine the cellular mechanisms underlying ischemic-type injury of premyelinated central axons.
\end{abstract}

Methods: Two thirds of axons in the post-natal day 10 rat optic nerve are small premyelinated axons $(<0.4 \mu \mathrm{m}$ diameter), and one third have undergone radial expansion in preparation for glial contact and the onset of myelination. Compound action potential recording and quantitative electron microscopy were used to examine the effect of modelled ischemia (oxygen-glucose deprivation) upon these two axon populations. Glutamate receptor expression was investigated using PCR and immuno-staining approaches at the confocal light and ultrastructural levels.

Results: Oxygen-glucose deprivation produced action potential failure and focal Deleted: Ischemia breakdown of the axolemma of small premyelinated axons at sites of contact with oligodendrocyte processes, which were also disrupted. The resulting axon loss was $\mathrm{Ca}^{2+}$ | dependent, $\mathrm{Na}^{+}$- and $\mathrm{Cl}^{-}$-independent and required activation of NMDA and non-NMDA glutamate receptors. NMDA receptor expression was localized to oligodendrocyte processes at sites of contact with premyelinated axons, in addition to expression within compact myelin, No peri-axonal NMDA receptor expression was observed on oligodendrocyte processes ensheathing large premyelinated axons and no protective effect of glutamate receptor block was observed in these axons.

Interpretation: NMDA receptor-mediated injury to oligodendrocyte processes navigating | along small premyelinated axons precedes damage to the underlying axon, a phenomena Deleted: itself Deleted: populations of axons. Expression of glutamate receptors Deleted: both that is lost following radial expansion and subsequent oligodendrocyte ensheathment. 


\section{Introduction}

Selective injury of developing white matter is the most frequent cause of cerebral palsy, the most common human birth disorder. The underlying pathology (periventricular leukomalacia: PVL), may feature focal necrotic damage of periventricular white matter and diffuse white matter injury. White matter injury may arise following hypoxiaischemia at birth, but the largest patient cohort suffer injury during mid-gestation ${ }_{-}^{1,2}$. The motor, sensory and cognitive deficits that characterize the lesion are associated with

Deleted: PVL

Deleted:

Field Code Changed injury to immature cells of the oligodendroglial lineage, which are susceptible to a form | of non-NMDA-type glutamate receptor (GluR) -mediated excitotoxic injury ${ }^{3-8}$. In addition to non-NMDA receptors, these cells express NMDA GluRs selectively on their processes $^{9-11}$, although the role of NMDA receptors in ischemic injury of myelinated white matter is controversial ${ }^{12-15}$.

Oligodendroglial injury has been the focus of research into the cellular mechanisms of developing white matter injury for the past decade. However, axon damage is consistently reported in histological examinations of PVL 2,16,17. Axonal changes include truncation of the axon cylinder ${ }^{18}$ and the formation of "retraction balls and clubs"16. Recent reports indicate that axonal injury is an early event in the formation of both focal and diffuse components of PVL ${ }^{19-21}$, and may be central to the evolution of the injury ${ }^{17}$. Despite these considerations, almost nothing is known regarding the injury mechanisms operating upon premyelinated central axons. In mid-gestation telencephalic, white matter that is subject to PVL, axons have an immature expression profile for developmental markers and oligodendroglia are changing from the precursor to the immature form ${ }^{22,23}$. The first evidence of early myelination is apparent, which includes myelin basic protein expression around some axons of the optic radiation ${ }^{23}$. There is evidence that injury to these axons during PVL can result in optic nerve hypoplasia ${ }^{24}$. Central axon development has been studied in detail in the rat optic nerve (RON), where radial expansion corresponding to an $\sim 3$ fold increase in diameter within an $\sim 4$ day period precedes first contact by oligodendrocyte processes and the subsequent onset of myelination 25 . The first post-mitotic oligodendrocytes appear at post-natal day 6 (P6) and start to extend processes parallel to axons ${ }^{26-28}$. The first ensheathed axons appear at this

Deleted: $\mathrm{PVL}^{2,16,17}$

Deleted: clubs" ${ }^{16}$. Recent reports
indicate that axonal injury is an early
event in the formation of both focal and
diffuse components of PVL ${ }^{19-21}$, and may
be central to the evolution of the injury ${ }^{17}$.
Despite these considerations, almost
nothing is known regarding the injury
mechanisms operating upon
premyelinated central axons. In mid-
gestation telencephalic and periatal white
matter that is subject to PVL, axons have
an immature expression profile for
developmental markers and
oligodendroglia are changing from the
precursor to the immature form ${ }^{22,23}$

Deleted: myelination ${ }^{25}$ 
age and correspond to the largest premyelinated axons with diameters of $>=0.4 \mu \mathrm{m}^{25}$. By P10 the first wraps of compact myelin appear, corresponding to $\sim 1 \%$ of axon profiles with $\sim 4 \%$ of axons ensheathed but not myelinated ${ }^{25}$, at which point the axons become sensitive to acute ischemic injury ${ }^{29}$. We here examine the mechanisms of acute ischemictype injury at this crucial developmental point and test the significance of GluR expression in the injury process.

\section{Methods}

All animal procedures conformed to UK home office regulations. RONs were dissected from Lister-hooded rats between P8-12. Nerves were perfused with artificial cerebrospinal fluid (aCSF), composition (in $\mathrm{mM}$ ): $\mathrm{NaCl}, 126 ; \mathrm{KCl}, 3 ; \mathrm{NaH}_{2} \mathrm{PO}_{4}, 2$; $\mathrm{MgSO}_{4}, 2 ; \mathrm{CaCl}_{2}, 2 ; \mathrm{NaHCO}_{3}, 26$; glucose, $10 ; \mathrm{pH}, 7.45$, bubbled with $5 \% \mathrm{CO}_{2} / 95 \% \mathrm{O}_{2}$ and maintained at $37^{\circ} \mathrm{C}$. Zero- $\mathrm{Na}^{+} \mathrm{aCSF}: \mathrm{NMDG} / \mathrm{Cl}, 124 ; \mathrm{KCl}, 3 ; \mathrm{CaCl}_{22} 2$; $\mathrm{MgSO}_{4}, 2$; choline- $\mathrm{HCO}_{3}, 26 ; \mathrm{KH}_{2} \mathrm{PO}_{4}$, 2; Glucose 10. Zero-Cl ${ }^{-}$aCSF: Na-cyclamate, 124; Kgluconate, 3; Ca gluconate, $2 ; \mathrm{MgSO}_{4}, 2 ; \mathrm{NaHCO}_{3}, 26 ; \mathrm{NaH}_{2} \mathrm{PO}_{4}, 2$; Glucose 10. Zero$\underline{\mathrm{Ca}^{2+}}$ aCSF: $\mathrm{CaCl}_{2}$ was omitted and 50 EGTA $\mu \mathrm{M}$ was added. Glucose was omitted from solutions when oxygen-glucose deprivation (OGD) was required and the solution was pre-bubbled with $5 \% \mathrm{CO}_{2} / 95 \% \mathrm{~N}_{2}$ for at least 60 min. The chamber atmosphere was switched to $5 \% \mathrm{CO}_{2} / 95 \% \mathrm{~N}_{2}$ during perfusion with OGD. Osmolarity of all solutions was measured and adjusted using $\mathrm{NaCl}$ or sucrose as required. Data are mean $\pm \mathrm{SEM}$, significance determined by t-test or ANOVA as appropriate. GluR antagonists were from Tocris (UK), all other reagents were from Sigma (UK) unless stated otherwise.

Electrophysiological recordings used the same equipment to that described | recently ${ }^{30}$. In brief, extracellular compound action potentials (CAPs) were evoked and recorded with suction electrodes. Peak-to-peak amplitude was used to assess changes in the number of unitary action potentials since CAP area can not be applied reliably to recordings from neonatal nerves due to the long stimulus duration required for supramaximal stimulation 29 . CAPs were evoked via square-wave constant current pulses of 150-600 $\mu \mathrm{sec}$ (Iso stim A320, WPI). The signal was amplified (Cyber Amp 320, Axon
Deleted: $\mathrm{Ca}_{2} \mathrm{Cl}$,

Deleted: $\mathrm{Ca}_{2}$-gluconate,

Deleted: zero- $\mathrm{Ca}^{2+}$
Deleted:

Field Code Changed

Deleted: times

Deleted:

Field Code Changed 
| Instruments), subtracted from a parallel differential electrode, filtered (low pass: $800_{z} \mathrm{~Hz}$ ), digitized (25000 Hz: 1401 mini, Cambridge Electronic Design) and displayed on a PC running Signal software (Cambridge Electronic Design). CAP loss is taken to indicate | irreversible failure of axon function ${ }^{29}$, and is well correlated with pathological changes in axons such as dissolution of microtubules/neurofilaments, disruption of mitochondria, loss of axoplasmic integrity and of neurofilament staining.

For immuno-histochemistry, P10 RONs were dissected into 0.1M PBS and fixed in $4 \%$ paraformaldehyde for $30 \mathrm{~min}$. Nerves were subsequently incubated in $0.1 \mathrm{M}$ PBS plus $20 \%$ sucrose w/v for $5 \mathrm{~min}$ prior to freeze-sectioning ( $20 \mu \mathrm{m}$ sections) and subsequent blocking for $60 \mathrm{~min}$ in $0.1 \mathrm{M}$ PBS, $10 \%$ fetal goat serum plus $0.5 \%$ Triton-X 100. Sections were then incubated in this solution plus primary antibody at $4^{\circ} \mathrm{C}$ overnight. Antibodies raised against NR1 (1:200, Upstate), GluR4 (1:200, Upstate), neurofilament-200 (NF-H), neurofilament-70 (NF-L) (Chemicon; 1:200 and 1:100 respectively), and CNPase (an oligodendroglial marker; Sigma; 1:100) were detected using appropriate Alexa-conjugated secondary antibodies (1:1000, Cambridge Bioscience, see ${ }^{10}$ for further details), and imaged as single planes via laser scanning confocal microscopy. Primary antibody omission controls were performed and are included in the figures at appropriate points. Intensity levels of neurofilament staining were assessed using mean pixel intensity levels within standard regions of interest, performed using Metamorph (Universal Imaging).

Deleted:

For PCR, P10 RONs were digested in MELT (Ambion) and subjected to a triazol extraction. RNA was then column purified and the resulting RNA treated to remove DNA contamination. The RNA was purified by extraction with phenol, $\mathrm{pH} 4.2$, (Sigma) and resuspended to a concentration of $1 \mu \mathrm{g} / \mu \mathrm{l}$. PCR was then performed using Omniscript (Qiagen) according to the manufacturer's instructions. All primers were tested on whole Deleted: brain as a positive control, see ${ }^{10}$ for further details.

For electron-microscopy, P10 RONs were collected at the end of electrophysiological experiments, washed in Sorenson's buffer and post-fixed in 3\% gluteraldehyde/Sorenson's. Nerves were then fixed with $2 \%$ osmium tetroxide and 
dehydrated prior to infiltration in epoxy. Ultrathin sections were counterstained with uranyl acetate and lead citrate and examined with a Jeol 100CX electron microscope. Glial cell processes could sometimes be identified on ultrastructural grounds ${ }_{v}^{6}$. For example, the astrocyte process in Fig 6 e contains characteristic wide-bore endoplasmic Deleted: Deleted: Fig reticulum and glycogen particles, and the oligodendrocyte process in Fig 4 e has myelinated a nearby axon. Since all axons in the adult rat optic nerve are myelinated, non-myelinated axons at $\mathrm{P} 10$ are in either an early or late stage of preparation for myelination. For post-embedding immuno-labelling of NR1 (1:100), GluR2/3 (1:50, Upstate) and KA2 (1:50, Upstate), primary antibody was applied to the sections overnight, and appropriate $20 \mathrm{~nm}$ gold particle secondary antibodies were applied following washing. Primary antibody omission controls were blank.

For morphometric analysis and viability scoring, axons within a minimum of three grid sections were outlined by hand using Image-J software (NIH) and axon area and perimeter were measured. Grids were selected at random and all identifiable axons within the area were included to avoid bias. Axon profiles were often far from round and the closest corresponding circular diameter was calculated from the perimeter values. Axon viability scores were assigned blind using the following scoring system. Axons were given one point for each of three well-established indicators of viability; i) the presence of a relatively complete axolemma, ii) the presence of normal microtubules, iii) the presence of a clear and debris-free axoplasm. Axons that showed all three of these features were therefore given a viability score of "3", and axons with none were given a score of "0", with intermediate scores between this range. Examples of axon scores are shown in supplemental Fig 1, where a healthy axon (score of "3"), and degrees of injury ("2" to "0") are shown.

Supplemental Figure 1 near here

\section{Results}


Action potential conduction in the isolated P10 RON was stable for several hours under control conditions, while $60 \mathrm{~min}$ of OGD resulted in conduction failure that was only partially reversible after 60 min recovery in $\operatorname{aCSF}_{n}^{29}(25.6 \pm 4.2 \%$ CAP recovery, $\mathrm{n}=14$ nerves, $\mathrm{P}<0.001$ vs. 130 min of control perfusion; Fig $1 \mathrm{a}$-c). Injury was largely prevented by removing extracellular $\mathrm{Ca}^{2+}(84.2 \pm 4.2 \%$ CAP recovery, $\mathrm{n}=8$ nerves,

\section{Deleted:}

Field Code Changed

Deleted: The injury process was largely negated

$\mathrm{P}<0.001$ vs. injury in normal $\left.\mathrm{Ca}^{2+} ; \mathrm{Fig} 1 \mathrm{a}-\mathrm{c}\right)$, an effect confirmed at the ultrastructural level (Fig 2 d). Staining for the axonal markers NF-L and NF-H, which label small diameter and larger diameter axons respectively $y_{z}^{31}$, was also disrupted in axons following

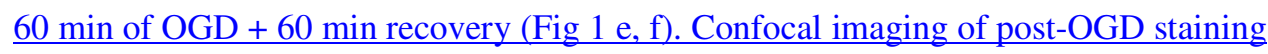
$\underline{\text { revealed areas denuded of neurofilament reactivity and ball-like structures that may }}$ correspond to swollen axon regions. Staining levels were quantified to reveal a similar decline in reactivity to NF-L and NH-H following the insult (Fig $1 \mathrm{f}$ ).

Figure 1 near here

1

Control perfused nerves had the ultrastructural features typical of this age $\mathbf{e}_{-\ldots, 27,28,}^{25,}$ 32. Small $(<0.4 \mu \mathrm{m}$ diameter $)$ and large $(=>0.4 \mu \mathrm{m})$ premyelinated axons where separated by a narrow extracellular space and by glial processes (Fig 2 a "S" and "L" premyelinated axons respectively), with the occasional myelinated axon apparent (Fig 2 a "Ax"). Some of the large premyelinated axons were ensheathed by glial processes (Fig $2 \mathrm{a}, " *$ "), shown in long-section in Fig $2 \mathrm{c}$ (arrow). Mitochondria in all cell compartments had a typical internal structure and showed no evidence of swelling. Following OGD, the extracellular space was expanded and nondescript cellular debris was common (Fig 2 b). Many premyelinated axons showed a loss of microtubules and regions of axolemma breakdown (Fig 2 b), and extensive disruption of glial processes was widespread (intact glial | processes were only occasionally seen), In long section, regions of intense focal premyelinated axon disruption were seen (Fig 2 e, "Axon" arrow heads), generally

Deleted: Note that the remaining identifiable axons in Fig 2 btend to be of large diameter $(=>0.4 \mu \mathrm{m})$. aligned parallel to damaged regions of neighbouring glial processes ("gp" arrow heads). Identification of axons in post OGD micrographs was based upon the presence of

\section{Deleted:}

Field Code Changed 
identifiable microtubules which were sometimes rather sparse (e.g., Fig 2 e "Axon" arrow).

(-

Figure 2 near here

Deleted: difficult. In Fig 2 e, the damaged axon had retained some microtubules ("Axon" arrow), permitting its identification (note the highergain).II

$\mid$

Unlike mature optic nerve axons $s_{-}^{33,34}$, replacing extracellular $\mathrm{Na}^{+}$(Fig 3 a-c;

supplemental Fig 2) or $\mathrm{Cl}^{-}$(Fig $3 \mathrm{~d}-\mathrm{f}$ ) was not protective against injury. This was not due to any toxicity of ion substitution at this age since control ion substitutions had no

significant non-reversible effect (Fig 3 a, c, d, f). This result suggests a fundamentally different ionic basis to injury in premyelinated and myelinated axons, and rules out a significant role for $\mathrm{Na}-\mathrm{Ca}$ exchange or $\mathrm{Cl}^{-}$channels in the injury process $\mathrm{x}_{--}^{33,34}$. In particular, $\mathrm{Na}^{+}$replacement leads to rapid depletion of axoplasmic $\mathrm{Na}^{+}$and block of reverse-mode $\mathrm{Na}-\mathrm{Ca}$ exchange, a major source of $\mathrm{Ca}^{2+}$ influx in mature myelinated axons during anoxia ${ }^{33}$. The finding was confirmed at the ultrastructural level where wide-scale disruption of axons following OGD in zero- $\mathrm{Na}^{+}$conditions was confirmed (supplemental

\section{Deleted:}

Deleted:

Field Code Changed

Fig 2). No changes were observed in the ultrastructure of nerves perfused with zero- $\mathrm{Na}^{+}$

aCSF for $140 \mathrm{~min}$ in the absence of OGD (not shown).

Figure 3 near here

Oligodendroglial GluRs are implicated in the $\mathrm{Ca}^{2+}$-dependent injury of their processes and somata $3,4,9-11$, while axonal expression of AMPA and kainate receptors is important for ischemic-type injury of myelinated spinal axons ${ }^{35,36}$. Block of NMDAreceptors (10 $\mu \mathrm{M}$ MK-801) or non-NMDA receptors (20 $\mu \mathrm{M} \underline{\text { NBQX) was protective }}$ against OGD-induced injury of premyelinated axons, as was combined block (67.9 $\pm 7.0 \%$ CAP recovery during combined block, $\mathrm{n}=8$ nerves, $\mathrm{P}<0.001$ vs. OGD alone; Fig 4 a-d). There was complete preservation of the small premyelinated axons during combined 
GluR block (Fig 4 e-f "s"), while large premyelinated axons showed lower levels of protection (Fig 4 e-g, "L"). Note in Fig $4 \mathrm{~g}$ that a large diameter premyelinated axon has lost all axoplasmic differentiation but a glial processes wrapped around the axon is intact (arrow heads).

Figure 4 near here

In control nerves, the diameter spectra of premyelinated axons ranged between $0.15-0.70 \mu \mathrm{m}$, with a peak between $0.25-0.30 \mu \mathrm{m}(\mathrm{n}=100$ axons; Fig $5 \mathrm{a}$, left $)$. This corresponds closely to earlier studies ${ }^{25}$. Following OGD, the density of recognizable premyelinated axons fell from $11.50 \mathrm{axons} / \mu \mathrm{m}^{2}$ to $1.26 \mathrm{axons} / \mu \mathrm{m}^{2}(\mathrm{P}<0.001 ; \mathrm{n}=91$ axons; Fig 5 a, right, filled bars). The loss of recognizable premyelinated axons was significantly reduced by GluR block (to 6.59 axons $/ \mu \mathrm{m}^{2}$ ), in particular in small premyelinated axons (Fig 5 a, right, open bars). The axon diameter spectra following OGD may be affected by expansion of the extracellular space and swelling of glial processes. To better assess the degree of injury within small and large premyelinated axons, we employed a numerical viability scoring system. The mean viability score for small premyelinated axons $(<0.4 \mu \mathrm{m}$ in diameter $)$ and large premyelinated axons $(=>0.4$ $\mu \mathrm{m}$ in diameter) are shown in Fig $5 \mathrm{~b}$. Under control conditions, the mean viability score of both groups of axons was close to "3", indicating no significant pathology. Following OGD the viability scores of both groups declined significantly, with the smaller axons showing a significantly greater decline than the larger axons $(\mathrm{P}<0.001)$. Combined GluR block prevented the appearance of pathology in identifiable small premyelinated axons but had no comparable effect upon the larger premyelinated axons. The viability scores for all premyelinated axons examined is shown in Fig 5 c-e, confirming the selective protection of small premyelinated axons by GluR block.

Figure 5 near here
Deleted: e-g "S"),

Deleted:

\begin{tabular}{|l|}
\hline Deleted: $0.1-0.7$ \\
\hline Deleted: in axon density \\
\hline Deleted: \\
\hline Field Code Changed \\
\hline
\end{tabular}


The pattern of NMDA receptor protein (Fig 6 a-c) and mRNA (Fig 6 d) expression was similar in P10 RON to that reported in the P10 mouse optic nerve ${ }_{-}^{10}$ Confocal imaging indicated that NR1 subunit protein is colocalized with CNPase (+) oligodendroglial processes but not with $\mathrm{N}-\mathrm{FH}(+)$ axon profiles (Fig $6 \mathrm{a}, \mathrm{b}$ ) GluR4 protein (non-NMDA subunit) appeared primarily in CNPase (+) oligodendroglial somata (Supplemental Fig 3). At the ultrastructural level, intense NR1 protein reactivity was found in regions where oligodendrocyte process membrane ran parallel to the axolemma of small premyelinated axons, in addition to expression within compact myelin as previously reported ${ }^{9,11}$ (Fig 6 e-g arrows, supplemental Figs 4, 5). NR1 reactivity appeared to translocate from the axolemmal face of oligodendrocyte processes aligned along small diameter premyelinated axons into the myelin as development progressed.

On no occasion was NR1 reactivity seen in the cell membrane of axons or astrocytes and it was unusual to see it in cell membranes of oligodendroglial somata.
Deleted: mRNA and

Deleted: nerve ${ }^{10}$, with clusters of NR1 NMDA subunit protein reactivity colocalized with CNPase (+) oligodendroglial processes but not with N-FH (+) axon profiles (Fig $6 \mathrm{a}, \mathrm{b}$ ). NonNMDA GluR

Deleted: , with clusters of NR1 NMDA subunit protein reactivity

Deleted: . Non-NMDA GluR

Deleted: subunits such as GluR4 colocalized primarily to

Deleted: 2).

Deleted: regions of

Deleted: were

Deleted: the cell membranes of

Deleted: processes

Deleted: in mature white matter

Field Code Changed

Deleted: occasion was NR1 reactivity seen in the cell membranes

Figure 6 near here

Deleted: $2-4$

Supplemental Figs 3-5 near here

Perfusion with AMPA+kainate produced a small but significant fall in the CAP

(Fig 7 a), which achieved higher significance in the presence of the AMPA

desensitization inhibitor cyclothiazide. Addition of NMDA to the test condition did not produce any significant augmentation of this effect (Fig 7 b "all agonists"). Immunolocalization using an antibody against the AMPA receptor Glur $2 / 3$ subunit revealed high expression in and under myelin and in the axolemma of premyelinated axons (Fig $7 \mathrm{c}-\mathrm{e}$ ).

The kainate receptor KA2 subunit showed more focal expression at sites within and under myelin, in addition to the axolemma of premyelinated axons (Fig $7 \mathrm{f}-\mathrm{i}$ ).

Figure 7 near here 


\section{Discussion}

Recent findings suggest that the intricate morphological arrangement that forms between axons and oligodendrocyte processes during central myelination is orchestrated in part by vesicular-type glutamate release from axons and subsequent detection by GluR on processes ${ }^{30,37,38}$. We have here shown NMDA receptor expression on oligodendrocyte processes, as required by this hypothesis. We have also shown that the presence of these NMDA receptors results in acute injury of small premyelinated axons during OGD. Possible mediators that might link NMDA receptor-mediated oligodendrocyte process injury with axon injury include local release of intracellular potassium resulting in axon depolarization and cytotoxic swelling; physical disruption of the axolemma during oligodendrocyte process swelling and dissolution, which will also disrupt the extracellular matrix; release of free radicals; and release of intracellular components such as calpains and lipases that are likely to attack the axon directly.

In addition to expression on oligodendrocyte processes, NMDA receptors were found in early compact myelin. Similar myelin expression in mature RON can mediate cytotoxic $\mathrm{Ca}^{2+}$ influx ${ }^{9-11}$. As a result, NMDA receptors contribute to OGD-induced injury in this preparation ${ }^{12}$, although apparently not in mouse optic nerve ${ }^{14,15}$. The current findings indicate that oligodendroglial processes express NMDA receptors where they navigate along premyelinated axons, while expression shifts to the myelin as it is deposited. NMDA receptor block failed to protect larger premyelinated axons, which is consistent with loss of receptors from the axonal face of oligodendrocyte processes once axon ensheathment has been achieved. At this point in central axon development, injury may involve the voltage-gate calcium channels transiently expressed in these large premyelinated axons ${ }^{30}$.

NMDA or AMPA/kainate receptor block was equally protective against OGDinduced injury. Combined block had no additional protective effect and resulted in sparing of small but not large premyelinated axons. Since oligodendrocyte NMDA receptors require co-activation of AMPA/kainate receptors to overcome $\mathrm{Mg}^{2+}$-block even
Deleted: The premyelinated central axons that are subject to injury during PVL are on the threshold of a program of rapid diameter expansion and axolemma protein re-organization. At this point in the P10 RON, oligodendroglia are maturing and extending processes parallel to axons as voltage-gated Na-channels start to cluster at sites where nodes of Ranvier will form ${ }^{26-28}$. Recent findings suggest that formation of the intricate morphological arrangement between axons and oligodendrocyte processes is dependent upon axonal vesicular-type glutamate release, which may co-ordinate axo-glial interactions via GluR on the oligodendrocyte processes ${ }^{30,35,36}$.

Premyelinated central axons also express functional glutamate transporters ${ }^{37}$, and vesicular- and transporter-mediated glutamate release are potential sources of the extracellular glutamate responsible for GluR-mediated injury of

oligodendrocyte processes described in

the current study. Astrocytes may also act as a source of ischemic glutamate release in developing white matter ${ }^{37,38}$. The

localization of NMDA receptors to

oligodendrocyte processes aligned along the axolemma of premyelinated axons indicates that $\mathrm{Ca}^{2+}$-dependent, $\mathrm{Na}^{+}$- and

$\mathrm{Cl}^{-}$-independent, injury to the processes at these sites precedes breakdown of the neighbouring axolemma. The findings therefore place oligodendrocyte pr ... [1]

Deleted: . Premyelinated central axons also express functional glutamate transporters ${ }^{37}$, and vesicular- and transporter-mediated glutamate release are potential sources of the extracellular glutamate responsible for GluR-mediated injury of oligodendrocyte processes

described in the current study. Astrocytes may also act as a source of ischemic glutamate release in developing white matter ${ }^{37,38}$. The localization of NMDA receptors to oligodendrocyte processes aligned along the axolemma of premyelinated axons indicates that $\mathrm{Ca}^{2+}$ dependent, $\mathrm{Na}^{+}$- and $\mathrm{Cl}^{-}$-independent, injury to the processes at these sites precedes breakdown of the neighbouring axolemma. The findings therefore place oligodendrocyte process injury before premyelinated axon injury in the

sequence of events that result in acute loss of function in early developing white matter during ischemia. II

In addition to expression on

oligodendrocyte processes aligned along premyelinated axons, NMDA receptor protein was also observed in early

compact myelin. Similar expression in myelin is seen in mature rat optic nerve where it can mediate cytotoxic $\mathrm{Ca}^{2+}$

influx ${ }^{9-11}$. Presumably as a result, NMDA receptors contribute to ischemic injury in myelinated rat optic nerve ${ }^{12}$, although no similar effect is found in mouse of ... [2] 
during modelled ischemia ${ }^{9}$, this is consistent with the current observation that both classes of GluR are present on oligodendrocyte processes. Immuno-labelling at the light level appears to show low AMPA/kainate receptor expression on oligodendrocyte processes ${ }^{10}$, but the current ultrastructural analysis reveals high levels of membrane expression in these structures in addition to clear expression in the axolemma. AMPA/kainate receptor activation produced $\sim 10 \%$ decline in the CAP, consistent with expression of functional receptors in the axolemma. Although a small contribution from AMPA/kainate receptors to axonal injury cannot be ruled out by the current findings, the absence of an additive effect of co-receptor block indicates that axonal non-NMDA GluRs are not directly involved in injury. This contrasts with the situation in mature central axons ${ }^{35,36}$, and may indicate that the axonal nanocomplexes incorporating AMPA/kainate receptors and NO synthase which are involved in acute OGD-injury have yet to form in premyelinated white matter.

The results may have significant clinical ramifications, since magnesium sulphate is a recognized antenatal intervention associated with a decreased risk of gross motor deficit in low birth weight babies ${ }^{39,40}$. While the significance of this effect requires further investigation, elevated extracellular $\mathrm{Mg}^{2+}$ will inhibit NMDA receptors in developing white matter ${ }^{9}$. The current findings raise the possibility that this prophylactic strategy may prove effective at protecting small premyelinated central axons in the foetus at risk of cerebral palsy, while interventions designed around NMDA receptor block ${ }^{41,42}$ may protect both oligodendroglial cells and their partner axons.

receptors in developing white matter ${ }^{9}$. The current findings raise the possibility that this prophylactic strategy may prove effective at protecting small premyelinated central axons in the foetus at risk of cerebral palsy, while interventions designed around NMDA receptor block ${ }^{41,42}$ may protect both oligodendroglial cells and their partner axons. 
Acknowledgements: We wish to thank Miss Natalie Allcock for technical assistance with electron microscopy and Mr Antonio Domingues for help with PCR. This work was supported by the National Institutes of Neurological Disorders and Stroke (NS44875). 
Figure Legends

Figure 1

OGD-induced injury in developing white matter. a: CAP recorded from the P10 RON before and $60 \mathrm{~min}$ after $60 \mathrm{~min}$ of OGD in normal- $\mathrm{Ca}^{2+}$ (left) and zero-Ca ${ }^{2+}$ (right) conditions. The times indicated correspond to the time after the start of each experiment. b: The mean action potential amplitude collapses during OGD and recovers partially, with greater recovery in zero- $\mathrm{Ca}^{2+}$ conditions. Note the stable CAP amplitude in aCSF ("Control"). c: Mean CAP recovery from OGD showing the protective effect of zero-Ca ${ }^{2+}$ (applied $20 \mathrm{~min}$ before and removed $40 \mathrm{~min}$ after OGD). d: Control experiment showing stable recording during perfusion with the zero- $\mathrm{Ca}^{2+}$ solution. e: Disruption of neurofilament staining following OGD. NF-L (left), and NF-H (right) staining in control nerve (top) is disrupted following OGD (bottom). Note the presence of swollen axonal | regions in the remaining NF-H (+) axons following OGD. f: The relative change in NF staining intensity between control and post-OGD nerves. "*" $=\mathrm{P}<0.05 ; " * * "=\mathrm{P}<0.01$. Scale bars $=10 \mu \mathrm{m}$.

Figure 2

Ultrastructural features of the injury induced by $60 \mathrm{~min}$ of OGD followed by $60 \mathrm{~min}$ recovery. a: Control perfusion for $120 \mathrm{~min}$. Small ("S") and large ("L") diameter premyelinated axons populate the RON, with occasional myelinated axons apparent ("Ax"). Some large premyelinated axons are ensheathed by glial processes (e.g., "*"), while other glial processes traverse the extracellular space between axons. Note that all axons contain microtubules and have intact axoplasmic membranes. Oligodendrocyte cell body indicated by "Oli". B: OGD + 60 min recovery. The extracellular space is greatly expanded and intact glial processes can no longer be identified. The number of | identifiable premyelinated axons is much reduced, c: Control long-section micrograph, Deleted: and consists mainly of larger illustrating the arrangement of oligodendrocyte processes (arrow) extending parallel to a 
premyelinated axon. d: Following OGD in zero- $\mathrm{Ca}^{2+}$, premyelinated axons have a normal appearance. e: Long section post-OGD. A small premyelinated axon retaining a small number of microtubules (arrow) has a disrupted axoplasm throughout the section shown and a region of intense focal damage (arrow heads). A glial process ("gp") aligned next to the axon is disintegrating at a similar point (arrow-heads). Scale bar a-d $=500 \mathrm{~nm}, \mathrm{e}=$

Deleted: $\mathrm{mm}$. $200 \mathrm{~nm}$.

Figure 3

The ionic-dependence of injury is novel. a (left): Representative CAP recorded before and after perfusion with zero- $\mathrm{Na}^{+}$solution, note the full recovery following washout. a (Right): Representative example of the absence of protection against OGD by zero- $\mathrm{Na}^{+}$ solution. Note the limited recovery following washout. b: Mean CAP amplitude, showing the low level of recovery following OGD in zero- $\mathrm{Na}^{+}$. Note that after $20 \mathrm{~min}$ of zero- $\mathrm{Na}^{+}$ perfusion the action potential is blocked. c: Data summary for zero- $\mathrm{Na}^{+}$experiments. $\mathrm{d}$ (left): Recordings before and after perfusion with zero- $\mathrm{Cl}^{-}$solution, note the full recovery following washout. $d$ (Right): Representative example of the absence of protection against $\mathrm{OGD}$ by zero- $\mathrm{Cl}^{-}$solution. Note the limited recovery following washout. e: Mean CAP amplitude, showing the low level of recovery following OGD in zero-Cl. f: Data summary from zero- $\mathrm{Cl}^{-}$experiments.

Figure 4

GluRs mediate premyelinated axon injury. a: NMDA receptor block (MK-801) increases mean CAP recovery following OGD. b, c: Block of non-NMDA GluR (NBQX) has a similar effect (b), as does combined application (c). d: The extent of recovery following these procedures. e, f: Small diameter premyelinated axons ("S") and oligodendrocyte processes ("op") are protected from OGD in the presence of MK-801 + NBQX, while larger axons ("L") are not. g: A large diameter premyelinated axon has no axoplasmic structures intact while a glial process wrapped around the axon is undamaged (arrow 
heads). "*" $=\mathrm{P}<0.05$; "***" $=\mathrm{P}<0.001$. Scale bars $=1 \mu \mathrm{m}$ in "e", $400 \mathrm{~nm}$ in "f", $100 \mathrm{~nm}$ in "g".

Figure 5

Morphometric analysis of OGD induced axon injury. a: The density of premyelinated axons (the number of axons in an area) of different diameter (the diameter that corresponds to the axon perimeter) present within the P10 RON. Left: Control nerve, | showing a peak in diameter between $0.25-0.3 \mu \mathrm{m}$ and a range between $0.15-0.7 \underline{0} \mu \mathrm{m}$. Right: The corresponding analysis for nerves exposed to OGD for $60 \mathrm{~min}$ followed by 60 min recovery (black bars). Note the much lower density of axons indicating loss of identifiable axons and expansion of the extracellular space. The corresponding analysis from nerves exposed to OGD in the presence of NBQX and MK-801 ("GluR block') shows that many identifiable axons are present (white bars). b: The mean axon viability score is shown for premyelinated axons below $0.4 \mu \mathrm{m}$ in diameter (black bars) and those of $0.4 \mu \mathrm{m}$ or above (white bars). Note that following OGD, both small and large premyelinated axons are significantly injured, with the small premyelinated axons effected more than the large premyelinated axons. Following OGD in the presence of GluR blockers only the small premyelinated axons are significantly damaged. c-e: Individual size spectra of the viability scores, showing greatly improved viability in axons $<0.4 \mu \mathrm{m}$ in the presence of GluR blockers. "***" $=\mathrm{p}<0.001$ vs. control. Deleted: $>0.4$

Figure 6

GluRs in developing white matter. a: NR1 (green, left) and CNPase (red, middle) appear to be co-localized (right) using fluorescent confocal imaging. Note that the NR1 clusters appear orange in the overlay. b: NR1 (green, left) and NF-H (red, middle) appear poorly co-localized (right). Note that the NR1 clusters appear green in the overlay. c: Absence of staining when NR1 antibody was omitted. d: mRNA for NMDA receptor subunits in P10 RON e-g: Localization of GluR NR1 subunit at the ultrastructural level by immuno-gold
Deleted: are

Deleted: are

Figure 7II 
labelling. e: A small diameter pre-myelinated axon ("s") is aligned between an astrocyte Deleted: a: process ("ap") and two myelinated axons ("Ax"). The boxed region is shown at higher Deleted: premyelinated axon ("S") | gain to the right. Three immuno-gold beads (short arrows) are present on the junction between an oligodendroglial process ("op") and the small premyelinated axon. Note the absence of NR1 reactivity on the astrocyte process. Also note the tubulovesicular complex apparently fusing with the axolemma at this point ("*"), and the NR1 reactivity Deleted: point. b: within the compact myelin of a neighbouring axon (short arrow). f: An oligodendrocyte somata ("Oli") is extending a process showing NR1 reactivity (hort arrows) along a small pre-myelinated axon. Note that no reactivity is seen in the somata itself. The boxed area is shown at higher gain to the right and reveals NR1 reactivity on the outer edge of an actively myelinating oligodendrocyte process. g: Two oligodendrocyte processes Deleted: C: (boxes) are navigating along small pre-myelinated axons (shown in great detail to the right). In both cases, NR1 reactivity is present on the axon side of the process. "gp" indicates that this glial process can not be positively identified as an oligodendrocyte process. Scale bars $=\underline{\mathrm{a}-\mathrm{c}:} 10 \mu \mathrm{m} ; \mathrm{e}-\mathrm{f}=500 \mathrm{~nm}$.

\section{Figure 7}

Non-NMDA GluRs are expressed in oligodendrocyte processes and the axolemma. a-b: The effect of GluR activation upon CAP amplitude in P10 rat optic nerve. a: Exposure to

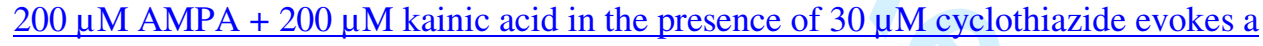
small reversible reduction in CAP amplitude. Sample CAPs are shown at the top, taken from the times indicated. Time "1" and "2" were used to calculate mean data for the histogram, revealing a significant reduction in amplitude in experiments performed in either the presence or the absence of cyclothiazide $(*=\mathrm{p}<0.05 ; * *=\mathrm{P}<0.01$ vs. control; ' $\mathrm{n}$ ' numbers as indicated). b: Exposure to $200 \mu \mathrm{M}$ AMPA $+200 \mu \mathrm{M}$ kainic acid $+1 \mathrm{mM}$ NMDA $+50 \mu \mathrm{M}$ glycine in the absence of $\mathrm{Mg}^{2+}$ also produced a small reversible fall in

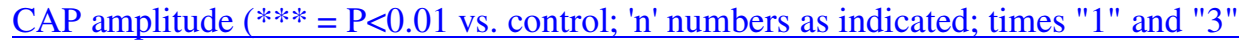
were used for the histogram data). Cyclothiazide has no effect applied alone. c-i: Localization of GluR 2/3 (c-e) and KA2 (f-i) subunit at the ultrastructural level by 
immuno-gold labelling. c: Strong staining (short arrows) is apparent for GluR 2/3 in a myelinating axon ("Ax"), with gold particles present within and under the myelin ("my"). The axon contains a mitochondria ("mit"). Staining is also present on the axoplasmic side of the axolemma (the axolemma is indicated by arrow heads) of a neighbouring small premyelinated axon ("s"). d: Gold particles largely located on the axoplasmic side of the myelin of a myelinating axon. e: Three gold particles are located on the interface between a glial processe ("gp") and a small pre-myelinated axon (axolemma indicated by arrowheads). f: Immuno-labelling for KA-2 reveals focal expression associated with the myelin of an actively myelinating axon. g: Focal axolemma staining of a premyelinated axon facing an ensheathing oligodendrocyte process ("op"). h: focal staining on the axolemma underneath an actively myelinating oligodendrocyte process. Note the near-by astrocyte processes ("ap"). i: KA-2 reactivity in the axoplasm and axolemma of small premyelinating axons contacted by glial processes. Scale $=500 \mathrm{~nm}$.

\section{Supplementary Figure 1}

Examples of the scoring system used for assessing axonal injury in premyelinated P10

RON axons. $\underline{\text { Scale bar }=1 \mu \mathrm{m}}$.

\section{Supplementary Figure 2}

Top: Micrograph showing the ultrastructural changes apparent in P10 RONs exposed to 60 min OGD in zero- $\mathrm{Na}^{+}$conditions. Examples of myelinated axons ("Ax") and small ("S") and large ("L") premyelinated axons can be seen. Note the widespread disruption of all axons and the typical "bubbling" of the inter-axonal face of the myelin. Scale bar = 1 Deleted: bars $=1 \mu \mathrm{m}$ $\underline{\mu \mathrm{m}}$. Bottom: Plot of CAP amplitude against time showing that prolonged reperfusion following OGD in zero-sodium conditions does not result in additional recovery. Mean CAP amplitude was $32.34 \pm 7.3 \%$ at $190 \mathrm{~min}(\mathrm{n}=5)$. 


\section{Supplementary Figure 3}

GluR subunit expression in P10 RON. a: CNPase(+) cells (red, left) and GluR4 (green, middle) co-localize (right). b: NF-H(+) axons (red, left) co-localize poorly with GluR4 (green; middle, right). c: NR1 (green, left) and GFAP (red, middle) expression are poorly co-localized (right). d: GluR4 (green, left) and GFAP (red, middle) expression are poorly co-localized (right). Scale bars $=10 \mu \mathrm{m}$.

\section{Supplementary Figure 4}

Immuno-electron micrograph showing NR1 reactivity in P10 RON. One contiguous oligodendrocyte process ("op") expressed NR1 reactivity at several sites that are close to small diameter premyelinated axons ("S"), including within extensions that appear to be

Deleted: axons. navigating along small axons (arrows).

\section{Supplementary Figure 5}

Immuno-electron micrographs showing NR1 reactivity in early compact myelin in P10 RON. Note that reactivity is present either within the myelin itself or on the outer surface as layers are being added. Scale bars $=500 \mathrm{~nm}$. 
References

1. Back SA, Rivkees SA. Emerging concepts in periventricular white matter injury. Semin Perinatol. 2004;28:405-414

2. Volpe JJ. Neurology of the newborn. Philadelphia: W.B. Saunders Company, 1995

3. Fern R, Moller T. Rapid ischemic cell death in immature oligodendrocytes: a fatal glutamate release feedback loop. J Neurosci. 2000;20:34-42

4. Follett PL, Rosenberg PA, Volpe JJ, Jensen FE. NBQX attenuates excitotoxic injury in developing white matter. J Neurosci. 2000;20:9235-9241

5. Back SA, Han BH, Luo NL et al. Selective vulnerability of late oligodendrocyte progenitors to hypoxia-ischemia. J Neurosci. 2002;22:455-463

6. Wilke S, Salter MG, Thomas R et al. Mechanism of acute ischemic injury of oligodendroglia in early myelinating white matter: the importance of astrocyte injury and glutamate release. J. Neurol. Exp. Neuropath. 2004;63:872-881

7. Matute C, Alberdi E, Ibarretxe G, Sanchez-Gomez MV. Excitotoxicity in glial cells. Eur J Pharmacol. 2002;447:239-246

8. Follett PL, Deng W, Dai W et al. Glutamate receptor-mediated oligodendrocyte toxicity in periventricular leukomalacia: a protective role for topiramate. $\mathrm{J}$ Neurosci. 2004;24:4412-4420

9. Karadottir R, Cavelier P, Bergersen LH, Attwell D. NMDA receptors are expressed in oligodendrocytes and activated in ischaemia. Nature.

2005;438:1162-1166

10. Salter MG, Fern R. NMDA Receptors are Expressed in Developing Oligodendrocyte Processes and Mediate Injury. Nature. 2005;438:1167-1171

11. Micu I, Jiang Q, Coderre $\mathrm{E}$ et al. NMDA receptors mediate calcium accumulation in myelin during chemical ischaemia. Nature. 2006;439:988-992

12. Bakiri Y, Hamilton NB, Karadottir R, Attwell D. Testing NMDA receptor block as a therapeutic strategy for reducing ischaemic damage to CNS white matter. Glia. 2008;56:233-240

13. McCarran WJ, Goldberg MP. White matter axon vulnerability to AMPA/kainate receptor-mediated ischemic injury is developmentally regulated. J Neurosci. 2007;27:4220-4229

14. Tekkok SB, Ye Z, Ransom BR. Excitotoxic mechanisms of ischemic injury in myelinated white matter. J Cereb Blood Flow Metab. 2007;27:1540-1552

15. Baltan S, Besancon EF, Mbow B et al. White matter vulnerability to ischemic injury increases with age because of enhanced excitotoxicity. J Neurosci. 2008;28:1479-1489

16. Banker B, Larrocher J-C. Periventricular leukomalacia of infancy: a form of neonatal anoxic encephalopathy. Arch Neurol. 1962;7:386-410

17. Dammann O, Hagberg H, Leviton A. Is periventricular leukomalacia an axonopathy as well as an oligopathy? Pediatr Res. 2001;49:453-457 
18. Marin-Padilla M. Developmental neuropathology and impact of perinatal brain damage. II: white matter lesions of the neocortex. J Neuropathol Exp Neurol. 1997;56:219-235

19. Haynes RL, Billiards SS, Borenstein NS et al. Diffuse Axonal Injury in Periventricular Leukomalacia as Determined by Apoptotic Marker Fractin. Pediatr Res. 2008

20. Hirayama A, Okoshi Y, Hachiya Y et al. Early immunohistochemical detection of axonal damage and glial activation in extremely immature brains with periventricular leukomalacia. Clin Neuropathol. 2001;20:87-91

21. Deguchi K, Oguchi K, Matsuura N et al. Periventricular leukomalacia: relation to gestational age and axonal injury. Pediatr Neurol. 1999;20:370-374

22. Haynes RL, Borenstein NS, Desilva TM et al. Axonal development in the cerebral white matter of the human fetus and infant. J Comp Neurol. 2005;484:156-167

23. Back SA, Luo NL, Borenstein NS et al. Late oligodendrocyte progenitors coincide with the developmental window of vulnerability for human perinatal white matter injury. J Neurosci. 2001;21:1302-1312

24. Jacobson L, Hellstrom A, Flodmark O. Large cups in normal-sized optic discs: a variant of optic nerve hypoplasia in children with periventricular leukomalacia. Arch Ophthalmol. 1997;115:1263-1269

25. Hildebrand C, Waxman SG. Postnatal differentiation of rat optic nerve fibers: electron microscopic observations on the development of nodes of Ranvier and axoglial relations. J Comp Neurol. 1984;224:25-37

26. Rasband MN, Peles E, Trimmer JS et al. Dependence of nodal sodium channel clustering on paranodal axoglial contact in the developing CNS. J Neurosci. 1999; 19:7516-7528

27. Skoff RP, Price DL, Stocks A. Electron microscopic autoradiographic studies of gliogenesis in rat optic nerve. II. Time of origin. J Comp Neurol. 1976;169:313334

28. Skoff RP, Price DL, Stocks A. Electron microscopic autoradiographic studies of gliogenesis in rat optic nerve. I. Cell proliferation. J Comp Neurol. 1976;169:291312

29. Fern R, Davis P, Waxman SG, Ransom BR. Axon conduction and survival in CNS white matter during energy deprivation: a developmental study. $\mathrm{J}$ Neurophysiol. 1998;79:95-105

30. Alix JJ, Dolphin AC, Fern R. Vesicular apparatus, including functional calcium channels, are present in developing rodent optic nerve axons and are required for normal node of Ranvier formation. J Physiol. 2008;586:4069-4089

31. Sanchez I, Hassinger L, Sihag RK et al. Local control of neurofilament accumulation during radial growth of myelinating axons in vivo. Selective role of site-specific phosphorylation. J Cell Biol. 2000;151:1013-1024

32. Vaughn JE. An electron microscopic analysis of gliogenesis in rat optic nerves. $Z$ Zellforsch Mikrosk Anat. 1969;94:293-324

33. Stys PK, Waxman SG, Ransom BR. Ionic mechanisms of anoxic injury in mammalian CNS white matter: role of $\mathrm{Na}+$ channels and $\mathrm{Na}(+)-\mathrm{Ca} 2+$ exchanger. J Neurosci. 1992;12:430-439 
34. Malek SA, Coderre E, Stys PK. Aberrant chloride transport contributes to anoxic/ischemic white matter injury. J Neurosci. 2003;23:3826-3836

35. Ouardouz M, Coderre E, Basak A et al. Glutamate receptors on myelinated spinal cord axons: I. GluR6 kainate receptors. Ann Neurol. 2009;65:151-159

36. Ouardouz M, Coderre E, Zamponi GW et al. Glutamate receptors on myelinated spinal cord axons: II. AMPA and GluR5 receptors. Ann Neurol. 2009;65:160-166

37. Ziskin JL, Nishiyama A, Rubio $M$ et al. Vesicular release of glutamate from unmyelinated axons in white matter. Nat Neurosci. 2007;10:321-330

38. Kukley M, Capetillo-Zarate E, Dietrich D. Vesicular glutamate release from axons in white matter. Nat Neurosci. 2007;10:311-320

39. Marret S, Doyle LW, Crowther CA, Middleton P. Antenatal magnesium sulphate neuroprotection in the preterm infant. Semin Fetal Neonatal Med. 2007;12:311317

40. Rouse DJ, Hirtz DG, Thom E et al. A randomized, controlled trial of magnesium sulfate for the prevention of cerebral palsy. N Engl J Med. 2008;359:895-905

41. Manning SM, Talos DM, Zhou C et al. NMDA receptor blockade with memantine attenuates white matter injury in a rat model of periventricular leukomalacia. J Neurosci. 2008;28:6670-6678

42. Fontaine RH, Olivier P, Massonneau V et al. Vulnerability of white matter towards antenatal hypoxia is linked to a species-dependent regulation of glutamate receptor subunits. Proc Natl Acad Sci U S A. 2008;105:16779-16784 
Page 11: [1] Deleted

Robert Fern

4/23/2009 1:20:00 PM

The premyelinated central axons that are subject to injury during PVL are on the threshold of a program of rapid diameter expansion and axolemma protein reorganization. At this point in the P10 RON, oligodendroglia are maturing and extending processes parallel to axons as voltage-gated Na-channels start to cluster at sites where nodes of Ranvier will form ${ }^{26-28}$. Recent findings suggest that formation of the intricate morphological arrangement between axons and oligodendrocyte processes is dependent upon axonal vesicular-type glutamate release, which may co-ordinate axo-glial interactions via GluR on the oligodendrocyte processes ${ }^{30,35,36}$. Premyelinated central axons also express functional glutamate transporters ${ }^{37}$, and vesicular- and transportermediated glutamate release are potential sources of the extracellular glutamate responsible for GluR-mediated injury of oligodendrocyte processes described in the current study. Astrocytes may also act as a source of ischemic glutamate release in developing white matter ${ }^{37,38}$. The localization of NMDA receptors to oligodendrocyte processes aligned along the axolemma of premyelinated axons indicates that $\mathrm{Ca}^{2+}$ dependent, $\mathrm{Na}^{+}$- and $\mathrm{Cl}^{-}$-independent, injury to the processes at these sites precedes breakdown of the neighbouring axolemma. The findings therefore place oligodendrocyte process injury before premyelinated axon injury in the sequence of events that result in acute loss of function in early developing white matter during ischemia.

In addition to expression on oligodendrocyte processes aligned along premyelinated axons, NMDA receptor protein was also observed in early compact myelin. Similar expression in myelin is seen in mature rat optic nerve where it can mediate cytotoxic $\mathrm{Ca}^{2+}$ influx ${ }^{9-11}$. Presumably as a result, NMDA receptors contribute to ischemic injury in myelinated rat optic nerve ${ }^{12}$, although no similar effect is found in mouse optic nerve ${ }^{14,15}$. The current findings indicate that NMDA receptor expression is high on oligodendroglial processes navigating along premyelinated axons but shifts to the myelin as it is deposited, possible becoming trapped in the lamella. The absence of protection of larger premyelinated axons by NMDA receptor block indicates that NMDA receptors are not restricted to the axonal face of the oligodendrocyte process membrane once it has ensheathed the axon cylinder. At this point in central axon development, injury may involve the voltage-gate calcium channels recently documented in this axon 
population ${ }^{30}$. The mechanism of small premyelinated axon injury following GluRmediated disintegration of neighbouring oligodendrocyte process will be difficult to dissect from the process injury itself. Possible mediators include local release of intracellular potassium onto the axolemma resulting in depolarization and cytotoxic swelling; physical disruption of the axolemma during oligodendrocyte process swelling and dissolution, which will also disrupt the extracellular matrix; release of free radicals capable of damaging the axolemma; and release of intracellular components such as calpains and lipases likely to attack the axon directly.

In addition to NMDA GluRs on their processes, developing oligodendroglia express non-NMDA receptors that can mediate toxic $\mathrm{Ca}^{2+}$-influx during ischemic conditions ${ }^{3-7}$. The current findings support the view that these receptors are expressed mainly on the somata ${ }^{10}$. Block of either NMDA or non-NMDA GluRs provided a similar degree of protection from ischemic conditions. This may reflect the significant degree of $\mathrm{Mg}^{2+}$-block of oligodendrocyte NMDA receptors ${ }^{9}$

$\begin{array}{lll}\text { Page 11: [2] Deleted } & \text { Robert Fern } & \mathbf{4} / \mathbf{2 3} / \mathbf{2 0 0 9} 1: \mathbf{2 0 : 0 0} \\ \text {. Premyelinated central axons also express functional glutamate transporters } & 37 \text {, and }\end{array}$ vesicular- and transporter-mediated glutamate release are potential sources of the extracellular glutamate responsible for GluR-mediated injury of oligodendrocyte processes described in the current study. Astrocytes may also act as a source of ischemic glutamate release in developing white matter ${ }^{37,38}$. The localization of NMDA receptors to oligodendrocyte processes aligned along the axolemma of premyelinated axons indicates that $\mathrm{Ca}^{2+}$-dependent, $\mathrm{Na}^{+}$- and $\mathrm{Cl}^{-}$-independent, injury to the processes at these sites precedes breakdown of the neighbouring axolemma. The findings therefore place oligodendrocyte process injury before premyelinated axon injury in the sequence of events that result in acute loss of function in early developing white matter during ischemia.

In addition to expression on oligodendrocyte processes aligned along premyelinated axons, NMDA receptor protein was also observed in early compact myelin. Similar expression in myelin is seen in mature rat optic nerve where it can 
mediate cytotoxic $\mathrm{Ca}^{2+}$ influx ${ }^{9-11}$. Presumably as a result, NMDA receptors contribute to ischemic injury in myelinated rat optic nerve ${ }^{12}$, although no similar effect is found in mouse optic nerve ${ }^{14,15}$. The current findings indicate that NMDA receptor expression is high on oligodendroglial processes navigating along premyelinated axons but shifts to the myelin as it is deposited, possible becoming trapped in the lamella. The absence of protection of larger premyelinated axons by NMDA receptor block indicates that NMDA receptors are not restricted to the axonal face of the oligodendrocyte process membrane once it has ensheathed the axon cylinder. At this point in central axon development, injury may involve the voltage-gate calcium channels recently documented in this axon population ${ }^{30}$. The mechanism of small premyelinated axon injury following GluRmediated disintegration of neighbouring oligodendrocyte process will be difficult to dissect from the process injury itself. Possible mediators include local release of intracellular potassium onto the axolemma resulting in depolarization and cytotoxic swelling; physical disruption of the axolemma during oligodendrocyte process swelling and dissolution, which will also disrupt the extracellular matrix; release of free radicals capable of damaging the axolemma; and release of intracellular components such as calpains and lipases likely to attack the axon directly.

In addition to NMDA GluRs on their processes, developing oligodendroglia express non-NMDA receptors that can mediate toxic $\mathrm{Ca}^{2+}$-influx during ischemic conditions ${ }^{3-7}$. The current findings support the view that these receptors are expressed mainly on the somata ${ }^{10}$. Block of either NMDA or non-NMDA GluRs provided a similar degree of protection from ischemic conditions. This may reflect the significant degree of $\mathrm{Mg}^{2+}$-block of oligodendrocyte NMDA receptors ${ }^{9}$, requiring cell depolarization via nonNMDA receptors before toxic $\mathrm{Ca}^{2+}$ influx can proceed. This may have significant clinical ramifications, since magnesium sulphate is a recognized antenatal intervention associated with a decreased risk of gross motor deficit in low birth weight babies ${ }^{39}$. While the significance of this effect requires further investigation, elevated extracellular $\mathrm{Mg}^{2+}$ will inhibit NMDA receptors in developing white matter ${ }^{9}$. The current findings raise the possibility that this prophylactic strategy may prove effective at protecting small premyelinated central axons in the foetus at risk of cerebral palsy. 


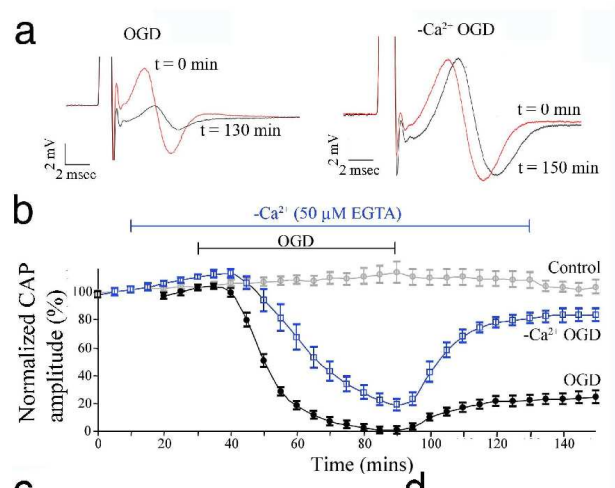

C
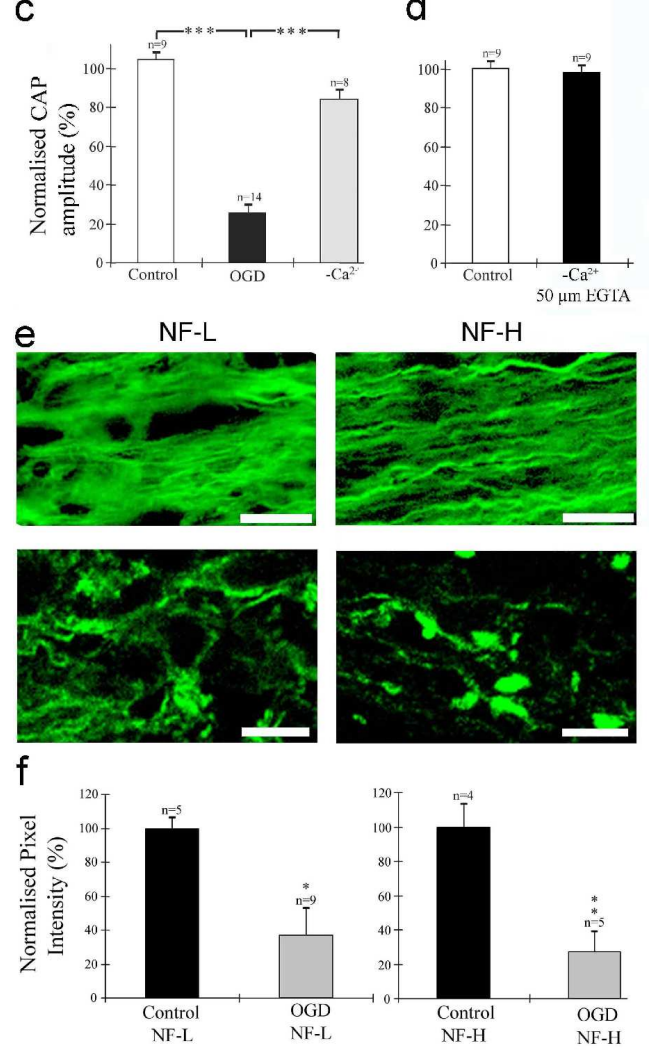

Figure 1

OGD-induced injury in developing white matter. a: CAP recorded from the P10 RON before and 60 min after $60 \mathrm{~min}$ of OGD in normal-Ca2+ (left) and zero-Ca2+ (right) conditions. The times indicated correspond to the time after the start of each experiment. $b$ : The mean action potential amplitude collapses during OGD and recovers partially, with greater recovery in zero-Ca2+ conditions. Note the stable CAP amplitude in aCSF ("Control"). c: Mean CAP recovery from OGD showing the protective effect of zero-Ca2+ (applied 20 min before and removed 40 min after OGD). $\mathrm{d}$ : Control experiment showing stable recording during perfusion with the zero-Ca2+ solution. e: Disruption of neurofilament staining following OGD. NF-L (left), and NF-H (right) staining in control nerve (top) is disrupted following OGD (bottom). Note the presence of swollen axonal regions in the remaining NF-H $(+)$ axons following OGD. $f$ : The relative change in NF staining intensity between control and post-OGD nerves. "*" $=\mathrm{P}<0.05 ; " * * "=\mathrm{P}<0.01$. Scale bars $=10 \mu \mathrm{m}$. 
$77 \times 190 \mathrm{~mm}(600 \times 600 \mathrm{DPI})$

John Wiley \& Sons 


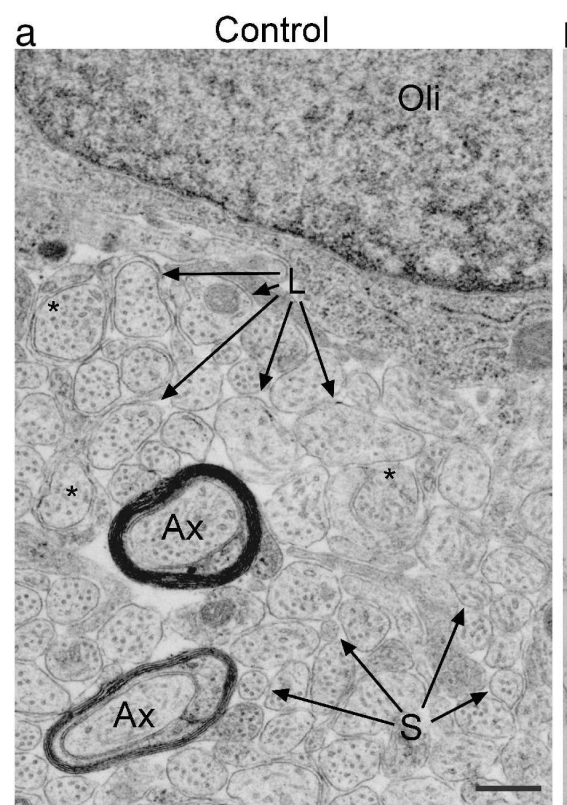

b OGD

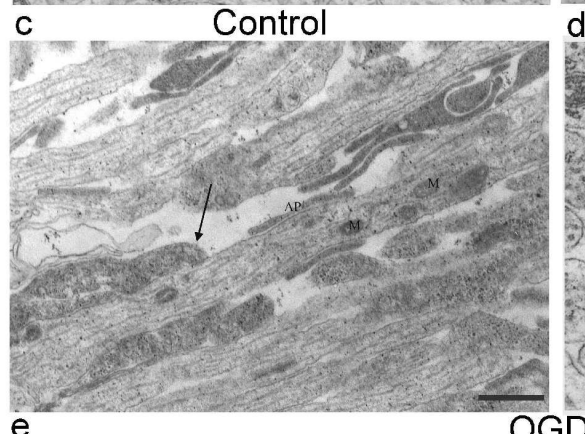

d $\quad O \mathrm{Ca}^{2+} \mathrm{OGD}$

e

OGD
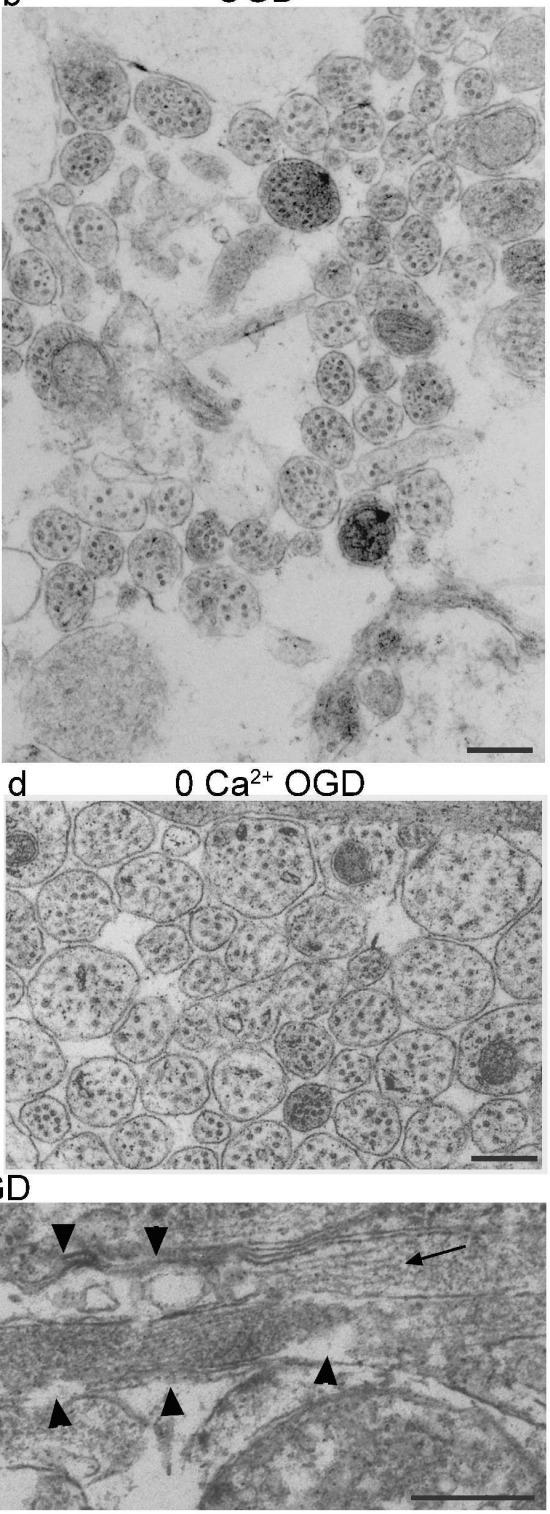

Figure 2

Ultrastructural features of the injury induced by 60 min of OGD followed by 60 min recovery. a: Control perfusion for $120 \mathrm{~min}$. Small ("S") and large ("L") diameter premyelinated axons populate the RON, with occasional myelinated axons apparent ("Ax"). Some large premyelinated axons are ensheathed by glial processes (e.g., "*"), while other glial processes traverse the extracellular space between axons. Note that all axons contain microtubules and have intact axoplasmic membranes. Oligodendrocyte cell body indicated by "Oli". B: OGD + $60 \mathrm{~min}$ recovery. The extracellular space is greatly expanded and intact glial processes can no longer be identified. The number of identifiable

premyelinated axons is much reduced. c: Control long-section micrograph, illustrating the arrangement of oligodendrocyte processes (arrow) extending parallel to a premyelinated axon. d: Following OGD in zero-Ca2+, premyelinated axons have a normal appearance. e: Long section postOGD. A small premyelinated axon retaining a small number of microtubules (arrow) has a disrupted 
axoplasm throughout the section shown and a region of intense focal damage (arrow heads). A glial process ("gp") aligned next to the axon is disintegrating at a similar point (arrow-heads). Scale bar $\mathrm{a}-\mathrm{d}=500 \mathrm{~nm}, \mathrm{e}=200 \mathrm{~nm}$.

$154 \times 215 \mathrm{~mm}(600 \times 600 \mathrm{DPI})$ 
$\mathrm{a}$
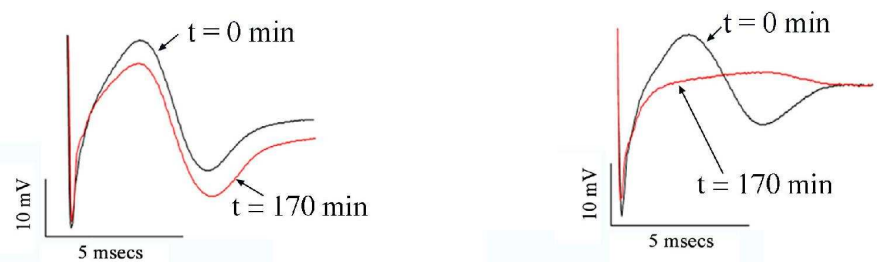

b

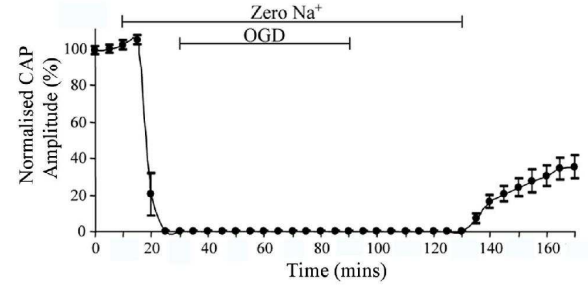

C

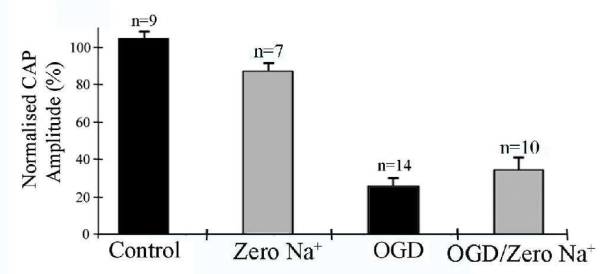

d
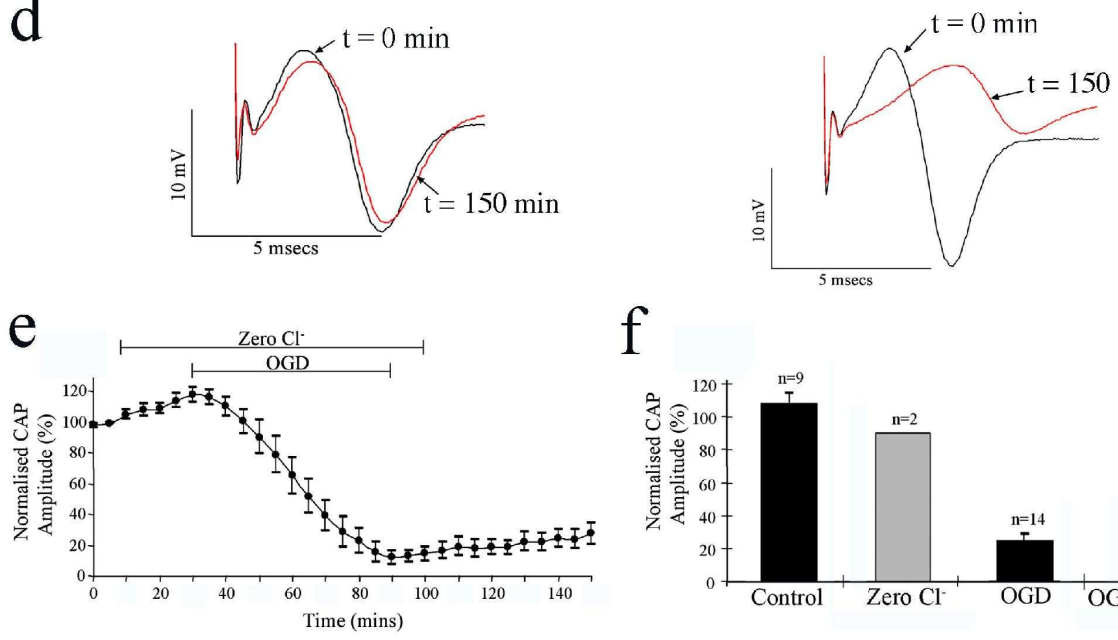

$f$

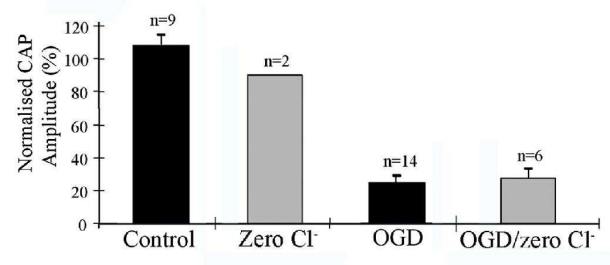

Figure 3

The ionic-dependence of injury is novel. a (left): Representative CAP recorded before and after perfusion with zero-Na+ solution, note the full recovery following washout. a (Right):

Representative example of the absence of protection against OGD by zero-Na+ solution. Note the limited recovery following washout. b: Mean CAP amplitude, showing the low level of recovery following OGD in zero-Na+. Note that after 20 min of zero-Na+ perfusion the action potential is blocked. c: Data summary for zero-Na+ experiments. $d$ (left): Recordings before and after perfusion with zero-Cl- solution, note the full recovery following washout. d (Right): Representative example of the absence of protection against OGD by zero-Cl- solution. Note the limited recovery following washout. e: Mean CAP amplitude, showing the low level of recovery following OGD in zero-Cl. f: Data summary from zero- $\mathrm{Cl}$ - experiments. 


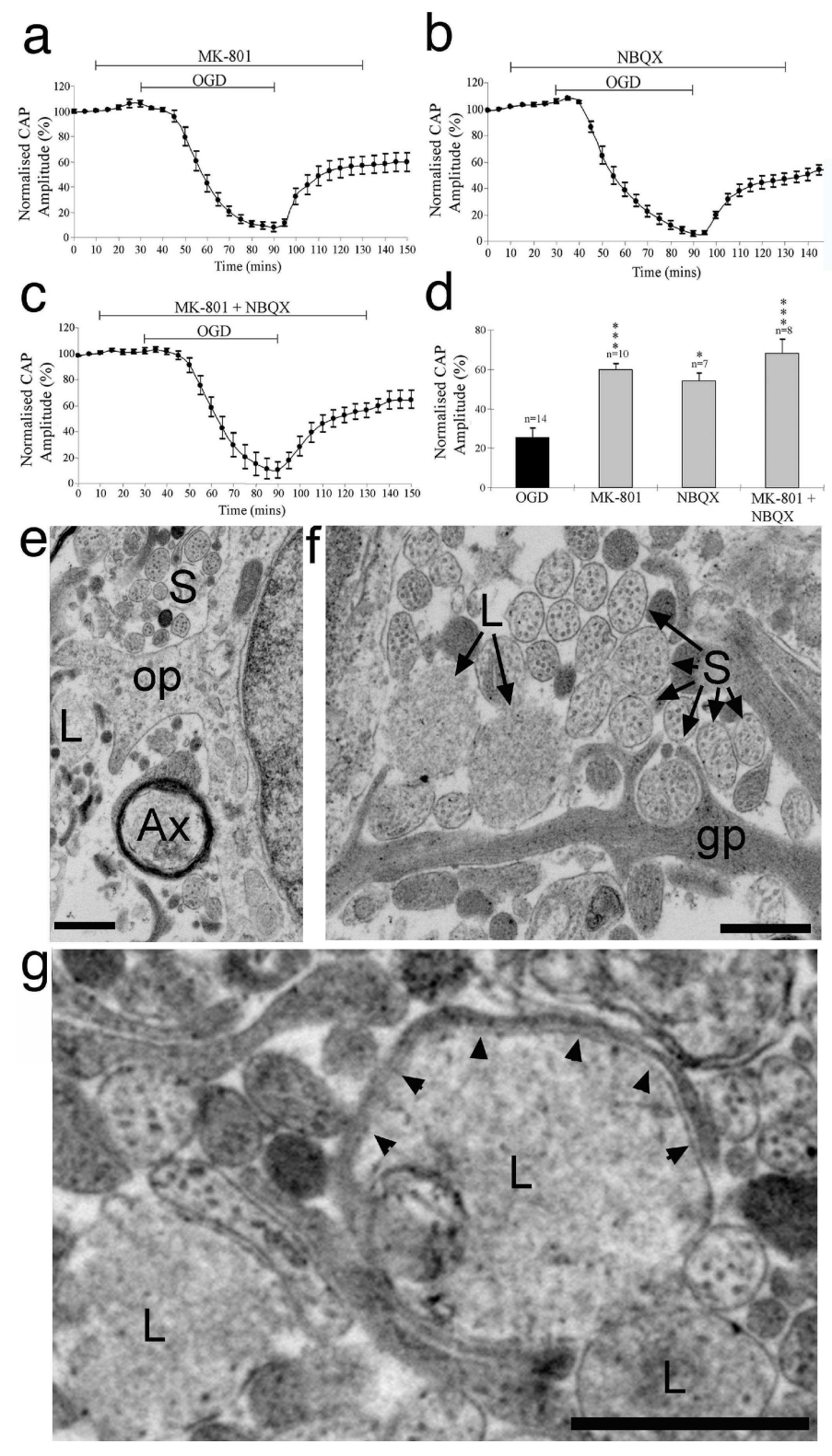

Figure 4

GluRs mediate premyelinated axon injury. a: NMDA receptor block (MK-801) increases mean CAP recovery following OGD. b, c: Block of non-NMDA GluR (NBQX) has a similar effect (b), as does combined application (c). d: The extent of recovery following these procedures. e, f: Small diameter premyelinated axons ("S") and oligodendrocyte processes ("op") are protected from OGD in the presence of MK-801 + NBQX, while larger axons ("L") are not. g: A large diameter premyelinated axon has no axoplasmic structures intact while a glial process wrapped around the axon is undamaged (arrow heads). " $* "=\mathrm{P}<0.05 ; " * * * "=\mathrm{P}<0.001$. Scale bars $=1 \mu \mathrm{m}$ in "e", $400 \mathrm{~nm}$ in "f", $100 \mathrm{~nm}$ in "g". 
 \\ Page 34 of 45 \\ Annals of Neurology}

John Wiley \& Sons 
a

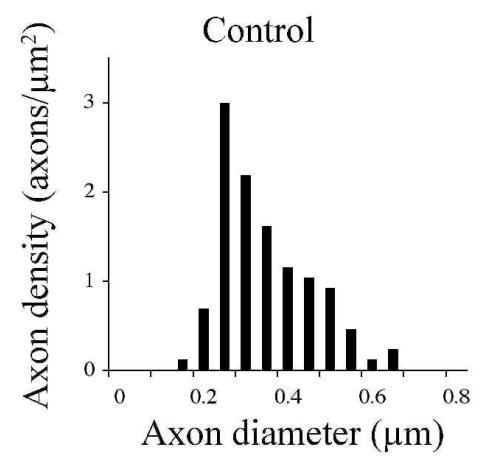

b

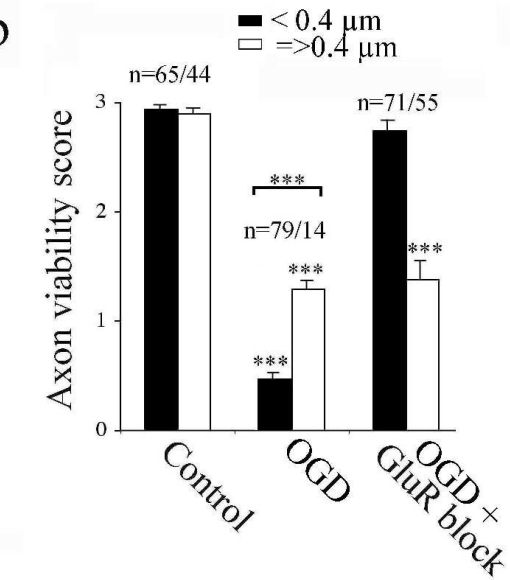

$\mathrm{d}$

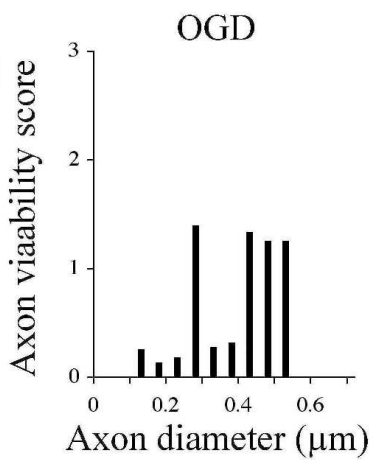

OGD

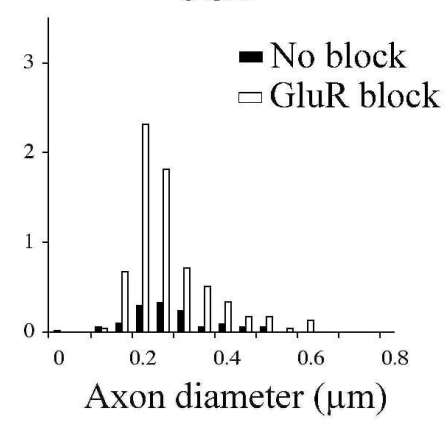

$\mathrm{C}$

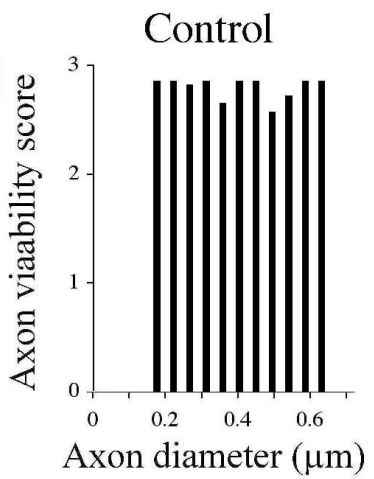

e

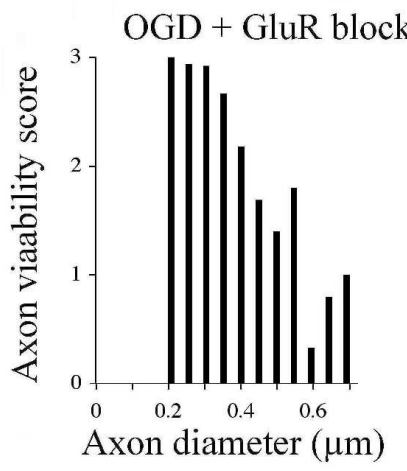

Figure 5

Morphometric analysis of OGD induced axon injury. a: The density of premyelinated axons (the number of axons in an area) of different diameter (the diameter that corresponds to the axon perimeter) present within the P10 RON. Left: Control nerve, showing a peak in diameter between 0.25-0.3 $\mu \mathrm{m}$ and a range between $0.15-0.70 \mu \mathrm{m}$. Right: The corresponding analysis for nerves exposed to OGD for 60 min followed by 60 min recovery (black bars). Note the much lower density

of axons indicating loss of identifiable axons and expansion of the extracellular space. The corresponding analysis from nerves exposed to OGD in the presence of NBQX and MK-801 ("GluR block') shows that many identifiable axons are present (white bars). b: The mean axon viability score is shown for premyelinated axons below $0.4 \mu \mathrm{m}$ in diameter (black bars) and those of $0.4 \mu \mathrm{m}$ or above (white bars). Note that following OGD, both small and large premyelinated axons are 
significantly injured, with the small premyelinated axons effected more than the large premyelinated axons. Following OGD in the presence of GluR blockers only the small premyelinated axons are significantly damaged. c-e: Individual size spectra of the viability scores, showing greatly improved viability in axons $<0.4 \mu \mathrm{m}$ in the presence of GluR blockers. "***" $=p<0.001$ vs. control.

$112 \times 179 \mathrm{~mm}(600 \times 600 \mathrm{DPI})$ 


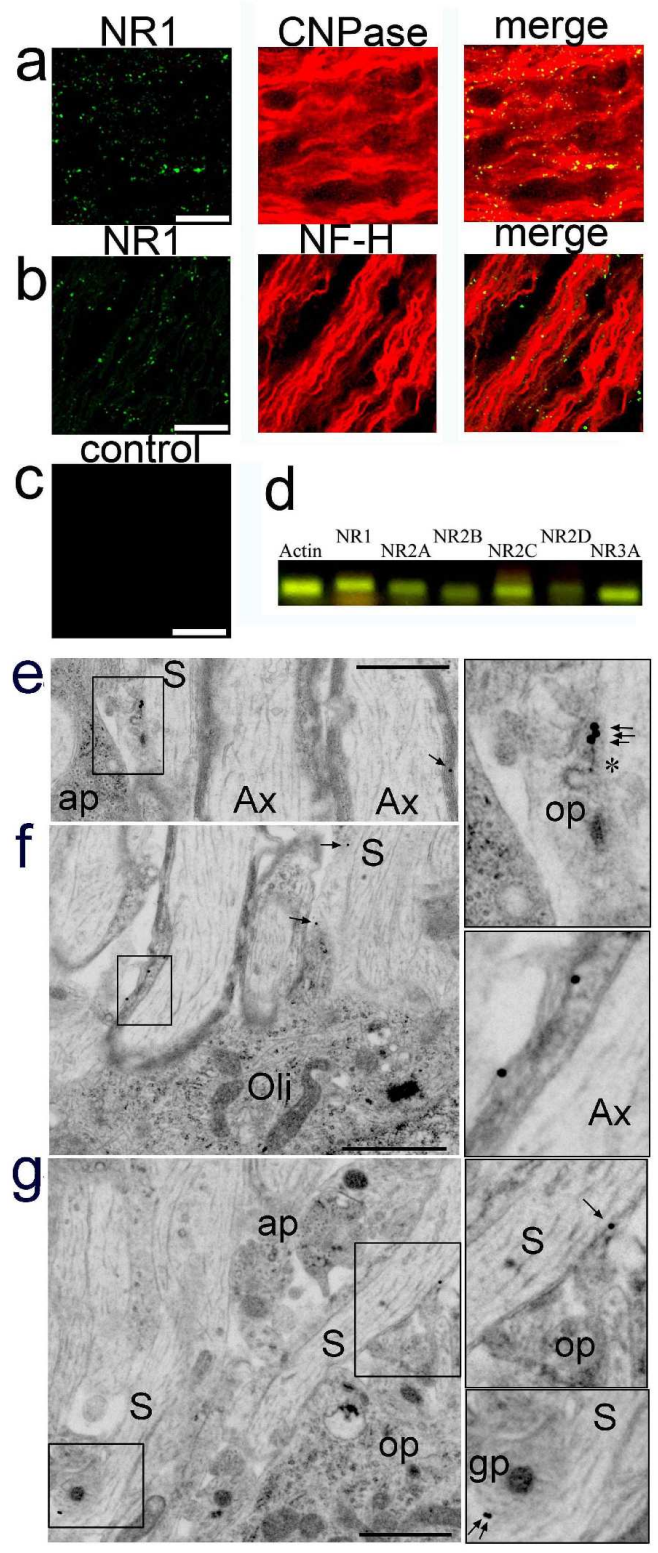

Figure 6

GluRs in developing white matter. a: NR1 (green, left) and CNPase (red, middle) appear to be colocalized (right) using fluorescent confocal imaging. Note that the NR1 clusters appear orange in the overlay. b: NR1 (green, left) and NF-H (red, middle) appear poorly co-localized (right). Note that the NR1 clusters appear green in the overlay. c: Absence of staining when NR1 antibody was omitted. d: mRNA for NMDA receptor subunits in P10 RON. e-g: Localization of GluR NR1 subunit at the ultrastructural level by immuno-gold labelling. e: A small diameter pre-myelinated axon ("s") is aligned between an astrocyte process ("ap") and two myelinated axons ("Ax"). The boxed region is shown at higher gain to the right. Three immuno-gold beads (short arrows) are present on the junction between an oligodendroglial process ("op") and the small premyelinated axon. Note the absence of NR1 reactivity on the astrocyte process. Also note the tubulovesicular complex apparently fusing with the axolemma at this point ("*"), and the NR1 reactivity within the compact 
myelin of a neighbouring axon (short arrow). f: An oligodendrocyte somata ("Oli") is extending a process showing NR1 reactivity (short arrows) along a small pre-myelinated axon. Note that no reactivity is seen in the somata itself. The boxed area is shown at higher gain to the right and reveals NR1 reactivity on the outer edge of an actively myelinating oligodendrocyte process. g: Two oligodendrocyte processes (boxes) are navigating along small pre-myelinated axons (shown in great detail to the right). In both cases, NR1 reactivity is present on the axon side of the process. "gp" indicates that this glial process can not be positively identified as an oligodendrocyte process. Scale bars = a-c: $10 \mu \mathrm{m} ; \mathrm{e}-\mathrm{f}=500 \mathrm{~nm}$.

$76 \times 182 \mathrm{~mm}(600 \times 600$ DPI $)$ 

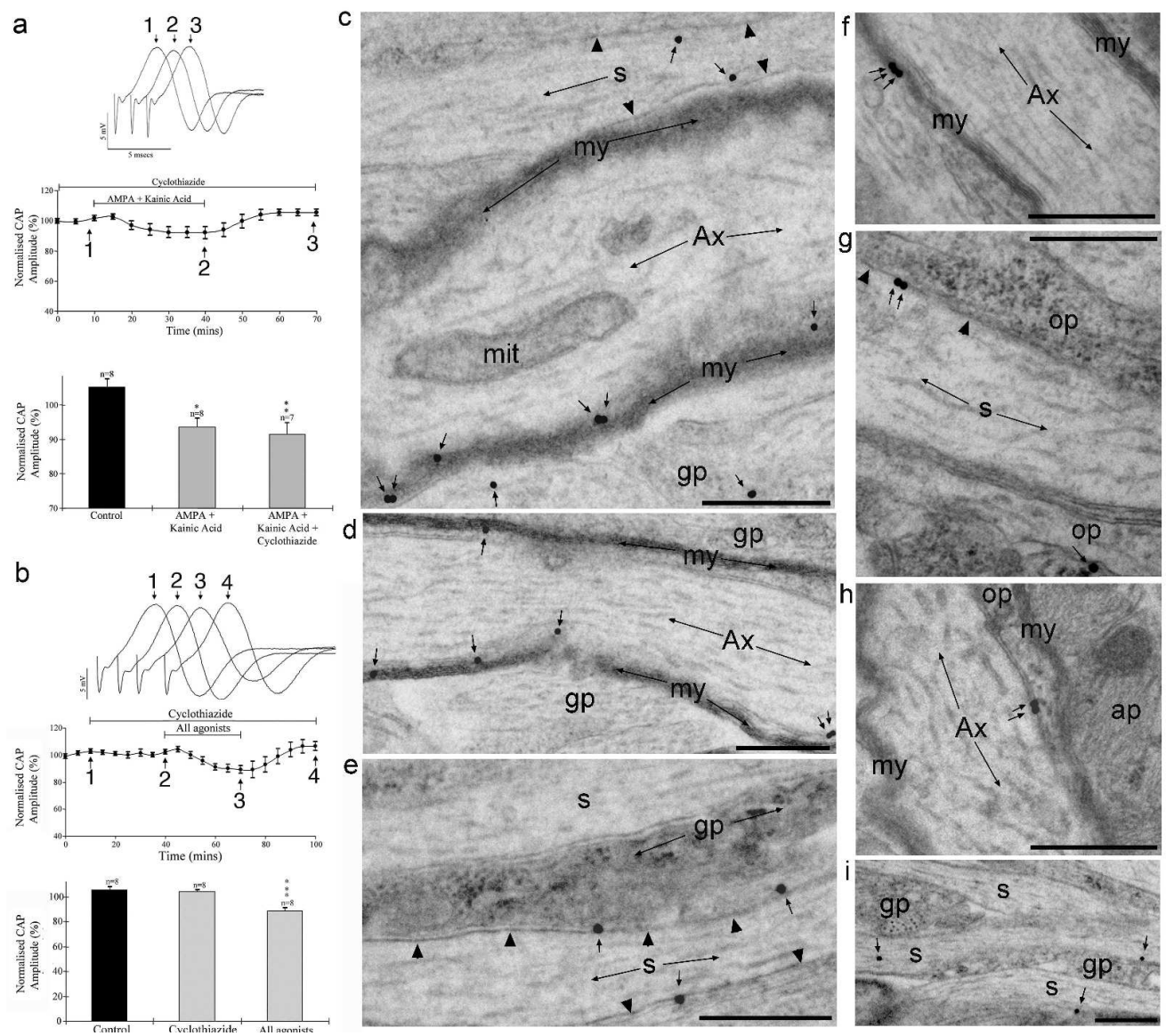

Figure 7

Non-NMDA GluRs are expressed in oligodendrocyte processes and the axolemma. a-b: The effect of GluR activation upon CAP amplitude in P10 rat optic nerve. a: Exposure to $200 \mu M$ AMPA $+200 \mu M$ kainic acid in the presence of $30 \mu \mathrm{M}$ cyclothiazide evokes a small reversible reduction in CAP amplitude. Sample CAPs are shown at the top, taken from the times indicated. Time "1" and "2" were used to calculate mean data for the histogram, revealing a significant reduction in amplitude in experiments performed in either the presence or the absence of cyclothiazide $(*=p<0.05 ; * *=$ $\mathrm{P}<0.01$ vs. control; ' $\mathrm{n}$ ' numbers as indicated). b: Exposure to $200 \mu \mathrm{M}$ AMPA $+200 \mu \mathrm{M}$ kainic acid + $1 \mathrm{mM} N M D A+50 \mu \mathrm{M}$ glycine in the absence of $\mathrm{Mg} 2+$ also produced a small reversible fall in CAP amplitude ( $* * *=P<0.01$ vs. control; ' $n$ ' numbers as indicated; times " 1 " and " 3 " were used for the histogram data). Cyclothiazide has no effect applied alone. c-i: Localization of GluR 2/3 (c-e) and KA2 ( $\mathrm{f}-\mathrm{i}$ ) subunit at the ultrastructural level by immuno-gold labelling. c: Strong staining (short arrows) is apparent for GluR 2/3 in a myelinating axon ("Ax"), with gold particles present within and under the myelin ("my"). The axon contains a mitochondria ("mit"). Staining is also present on the axoplasmic side of the axolemma (the axolemma is indicated by arrow heads) of a neighbouring small premyelinated axon ("s"). d: Gold particles largely located on the axoplasmic side of the myelin of a myelinating axon. e: Three gold particles are located on the interface between a glial processe ("gp") and a small pre-myelinated axon (axolemma indicated by arrowheads). f: Immunolabelling for KA-2 reveals focal expression associated with the myelin of an actively myelinating axon. g: Focal axolemma staining of a premyelinated axon facing an ensheathing oligodendrocyte process ("op"). h: focal staining on the axolemma underneath an actively myelinating

oligodendrocyte process. Note the near-by astrocyte processes ("ap"). i: KA-2 reactivity in the axoplasm and axolemma of small premyelinating axons contacted by glial processes. Scale $=500$ 
$\mathrm{nm}$.

$203 \times 179 \mathrm{~mm}(600 \times 600 \mathrm{DPI})$ 


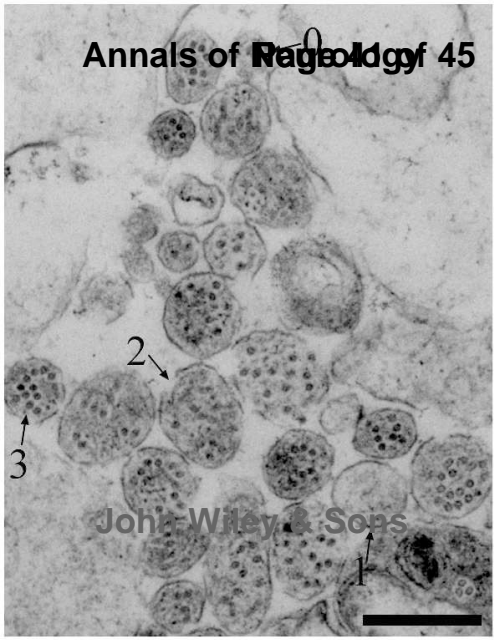



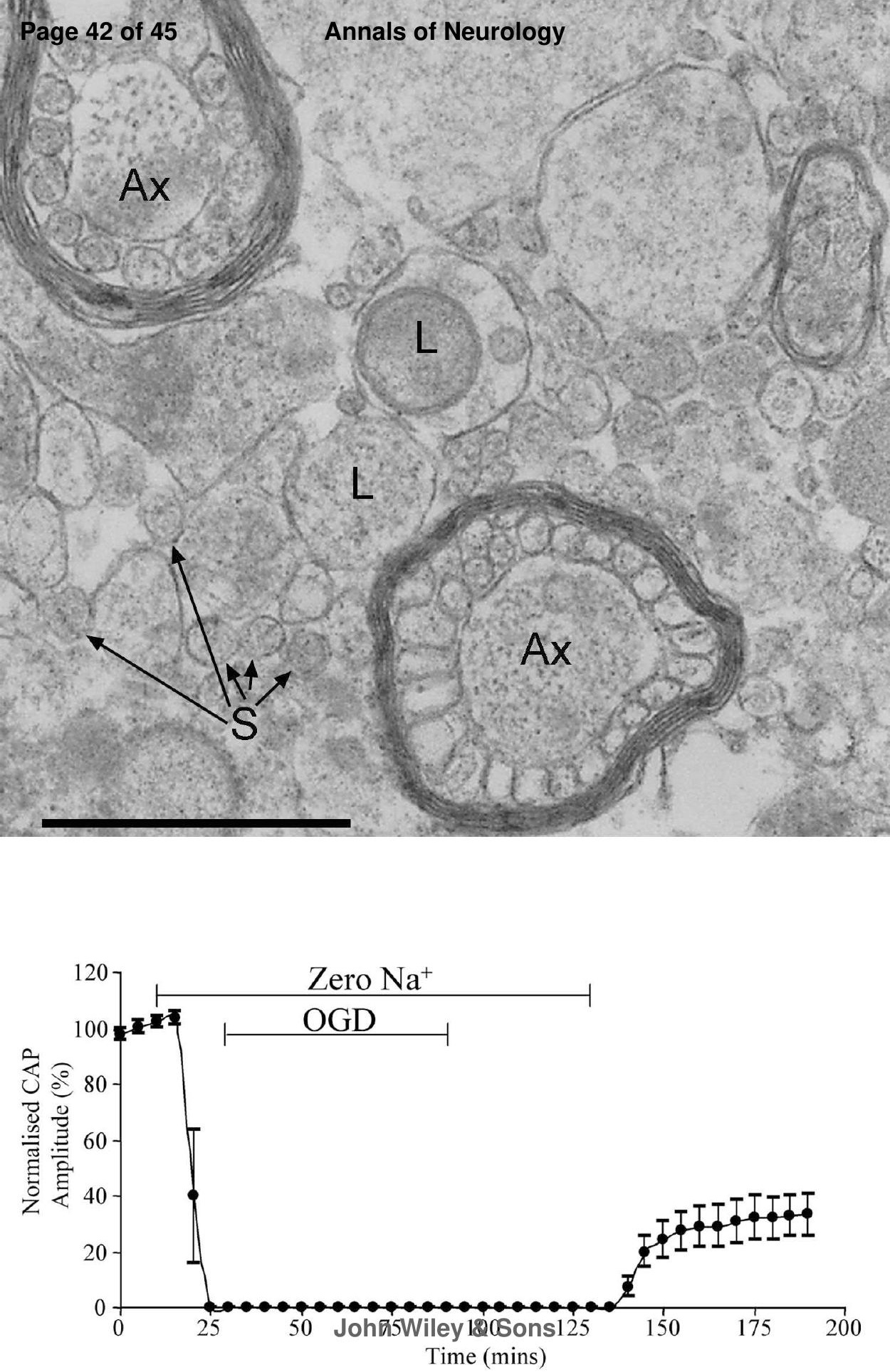


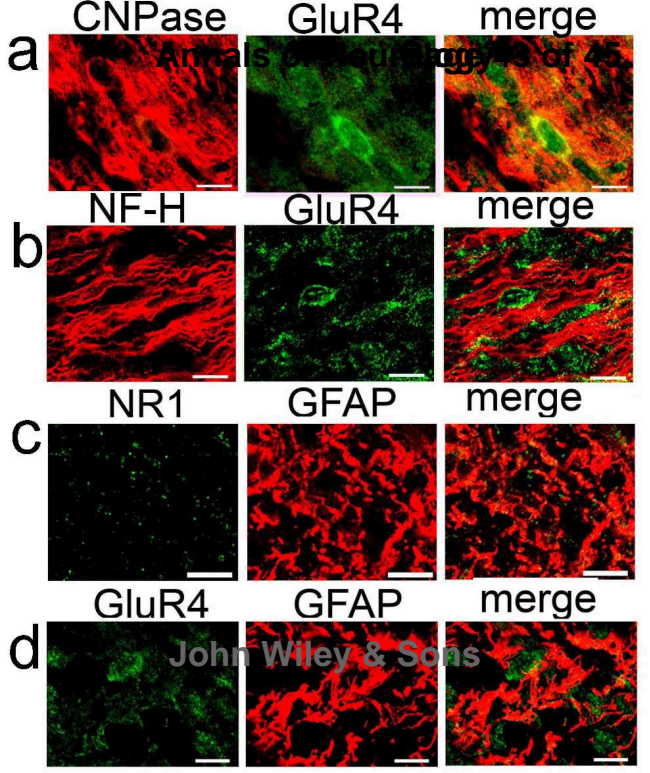




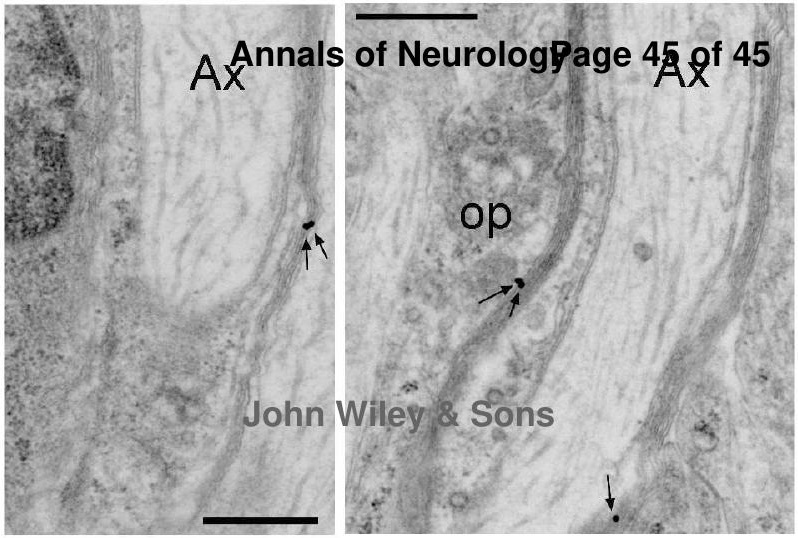

